\title{
Lithium-free transition metal monoxides for positive electrodes in lithium-ion batteries
}

\author{
Sung-Kyun Jung ${ }^{1,2}$, Hyunchul Kim ${ }^{3}$, Min Gee Cho ${ }^{2,4}$, Sung-Pyo Cho ${ }^{5}$, Byungju Lee ${ }^{1}$, Hyungsub Kim ${ }^{1,6}$, \\ Young-Uk Park', Jihyun Hong ${ }^{1}$, Kyu-Young Park ${ }^{1,2}$, Gabin Yoon ${ }^{1,2}$, Won Mo Seong', Yongbeom Cho', \\ Myoung Hwan $\mathrm{Oh}^{7}$, Haegyeom Kim ${ }^{1}$, Hyeokjo Gwon ${ }^{8}$, Insang Hwang', Taeghwan Hyeon ${ }^{2,4}$, \\ Won-Sub Yoon ${ }^{3 \star}$ and Kisuk Kang ${ }^{1,2 \star}$
}

\begin{abstract}
Lithium-ion batteries based on intercalation compounds have dominated the advanced portable energy storage market. The positive electrode materials in these batteries belong to a material group of lithium-conducting crystals that contain redoxactive transition metal and lithium. Materials without lithium-conducting paths or lithium-free compounds could be rarely used as positive electrodes due to the incapability of reversible lithium intercalation or the necessity of using metallic lithium as negative electrodes. These constraints have significantly limited the choice of materials and retarded the development of new positive electrodes in lithium-ion batteries. Here, we demonstrate that lithium-free transition metal monoxides that do not contain lithium-conducting paths in their crystal structure can be converted into high-capacity positive electrodes in the electrochemical cell by initially decorating the monoxide surface with nanosized lithium fluoride. This unusual electrochemical behaviour is attributed to a surface conversion reaction mechanism in contrast with the classic lithium intercalation reaction. Our findings will offer a potential new path in the design of positive electrode materials in lithium-ion batteries.
\end{abstract}

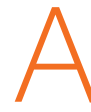
s lithium-ion batteries approach the energy density ceiling permitted by conventional intercalation compounds, high demand has arisen for new electrode materials ${ }^{1-3}$. The discovery of a positive electrode-a key performance component of lithium-ion batteries-with high energy/power density, low cost, and long cycle life will expedite progress in emerging areas such as electric vehicles and large-scale energy storage systems. Intercalation-based positive electrode materials contain transition metal ions (preferably early $3 d$ metals) as redox-active elements and lithium ions as charge carriers. Lithium ions diffuse in and out of the material during battery cycling, and thus the host should contain intrinsic lithium conduction paths. Generally, positive electrodes donate lithium ions in lithium-ion batteries, since metallic lithium and lithium-containing negative electrode materials present safety concerns and are chemically unstable ${ }^{4,5}$. Accordingly, the most commonly explored positive electrode materials are lithiumcontaining transition metal compounds with a so-called one-, twoor three-dimensional open structure, such as olivine $\left(\mathrm{LiMPO}_{4}\right.$; $\mathrm{M}=$ transition metal), layered $\left(\mathrm{LiMO}_{2}\right)$, or spinel $\left(\mathrm{LiM}_{2} \mathrm{O}_{4}\right)$ materials $^{6-8}$. Unfortunately, these material groups constitute only a tiny fraction of the existing redox-active transition metal compounds in nature. Numerous transition metal oxides, sulfides, phosphates and nitrides exist, some of which may exhibit suitable electrochemical potentials versus lithium as a positive electrode, which have been ignored since they are lithium-free or contain no lithium conduction path. Alleviating these restrictions would offer unexplored opportunities to identify new positive electrodes for lithium-ion batteries.
Here, it is shown that transition metal monoxides ( $M O, M=M n$, $\mathrm{Fe}, \mathrm{Co}$ ) that do not contain either intrinsic lithium or a lithium conduction path in their structure can nonetheless function as high-capacity positive electrode materials. These transition metal monoxides are known to be electrochemically active only in negative electrodes (lower than $\sim 1 \mathrm{~V}$ versus $\mathrm{Li}^{+} / \mathrm{Li}$ ) by the conversion reaction ${ }^{9}$. We demonstrate herein that when they are blended with nanosized lithium fluoride $(\mathrm{LiF})$ in the electrode, they not only donate lithium ions to the negative electrode but also exhibit an average voltage of over $3 \mathrm{~V}$ (versus $\mathrm{Li}^{+} / \mathrm{Li}$ ), functioning as a positive electrode. This study focuses on $\mathrm{MnO}$, although characteristics of $\mathrm{FeO}$ and $\mathrm{CoO}$ are also introduced. This new energy storage mechanism, investigated through a combination of experiment and first-principles calculation, expands our current understanding and brings a variety of unexplored materials into consideration as positive electrode materials.

\section{LiF-MO nanocomposite as positive electrode}

Mixtures of $\mathrm{LiF}$ and $\mathrm{MO}(\mathrm{M}=\mathrm{Mn}, \mathrm{Fe}, \mathrm{Co})$ were fabricated using a high-energy ball miller under an argon atmosphere (Fig. 1a). The presence of the mixture ( $\mathrm{LiF}$ and $\mathrm{MO}$ ) was confirmed using $\mathrm{X}$-ray diffraction (Fig. 1b) without any impurity phase, which may be formed during the mechanochemical mixing (Supplementary Figs 1 and 2$)^{10}$. The particle size of the composite was around $8 \mathrm{~nm}$ as shown by high-resolution transmission electron microscopy (TEM) (Supplementary Fig. 3). The mechanochemically treated $\mathrm{MnO} / \mathrm{C}$ composite was synthesized for comparison with the

\footnotetext{
${ }^{1}$ Department of Materials Science and Engineering, Research Institute of Advanced Materials (RIAM), Seoul National University, Seoul 08826, Republic of Korea. ${ }^{2}$ Center for Nanoparticle Research, Institute for Basic Science (IBS), Seoul 08826, Republic of Korea. ${ }^{3}$ Department of Energy Science (DOES), Sungkyunkwan University, Suwon 16419, Republic of Korea. ${ }^{4}$ School of Chemical and Biological Engineering and Institute of Chemical Processes, Seoul National University, Seoul 08826, Republic of Korea. ${ }^{5}$ National Center for Inter-University Research Facilities, Seoul National University, Seoul 08826, Republic of Korea. ${ }^{6}$ Korea Atomic Energy Research Institute (KAERI), 111 Daedeok-daero 989 Beon-Gil, Yuseong-gu, Daejeon 34057, Republic of Korea. ${ }^{7}$ Department of Chemistry, University of California, Berkeley, California 94720, USA. ${ }^{8}$ Department of Materials Science and Engineering, KAIST (Korea Advanced Institute of Science and Technology), Daejeon 34141, Republic of Korea. *e-mail: wsyoon@skku.edu; matlgen1@snu.ac.kr
} 
a

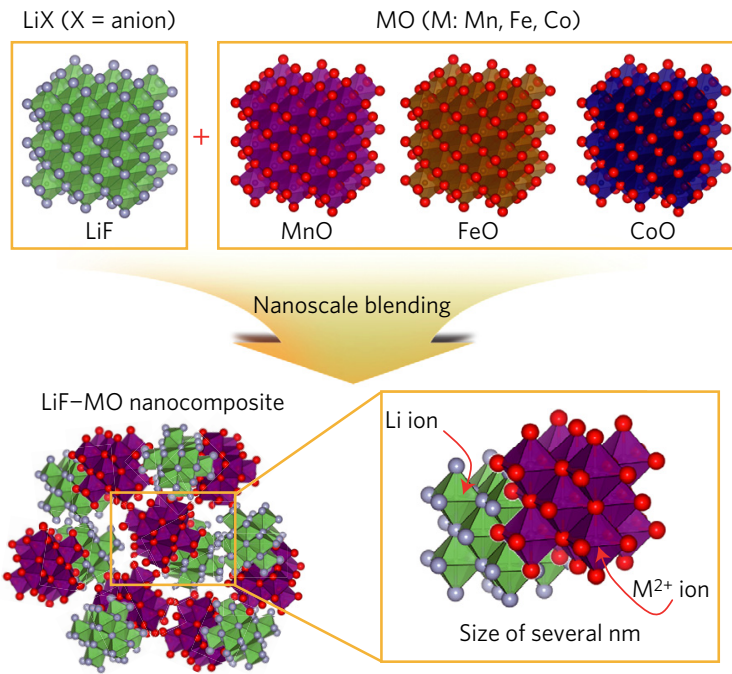

b

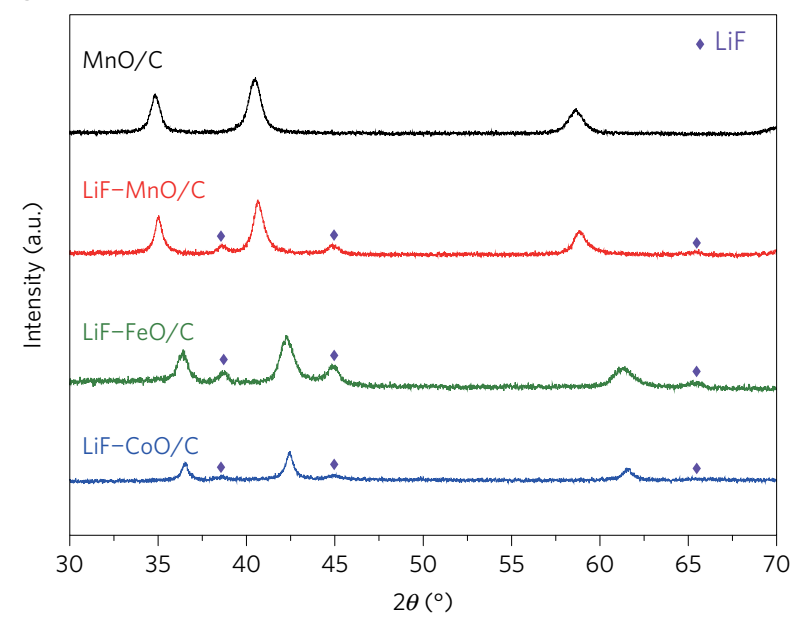

Figure 1 | Transition of metal monoxide from negative electrode to positive electrode material. a, Schematic of the positive electrode material design strategy using metal monoxide (MO). Metal monoxides that had electrochemical activity only as the negative electrode were designed as positive electrode material by forming mixtures with LiF in nanoscale under high-energy ball milling. After mechanochemical mixing, LiF and MO exist without chemical reaction in the several nanometre scale. The crystal structure of all compounds is described by a polyhedral image. Red, O; grey, $\mathrm{F}$; green, Li; purple, Mn; brown, Fe; blue, Co. b, Powder X-ray diffraction patterns of the MnO/C composite and LiF-MO (M = Mn, Fe, Co) nanocomposites.

LiF-MnO nanocomposite to verify the effect of the lithium compound ( $\mathrm{LiF})$.

$\mathrm{LiF}-\mathrm{MO}(\mathrm{M}=\mathrm{Mn}, \mathrm{Fe}, \mathrm{Co})$ nanocomposites showed positive electrode properties with high voltage and capacity after the first charging step (Fig. 2). Almost 0.9 of the lithium in the LiF-MnO nanocomposite participated in the reversible reaction (theoretical capacity corresponding to one lithium: $262.63 \mathrm{mAh} \mathrm{g}^{-1}$ ) at a $3.1 \mathrm{~V}$ average voltage. Meanwhile, the $\mathrm{MnO} / \mathrm{C}$ composite was nearly inactive in the voltage range $>1.5 \mathrm{~V}$ and had electrochemical activity only as the negative electrode from the conversion reaction within a low voltage range $(<1.0 \mathrm{~V})$ (Supplementary Fig. 4$)^{9,11-13}$. This implies that the addition of $\mathrm{LiF}$ critically affects $\mathrm{MnO}$ activation during the electrochemical reaction. The characteristic profile of $\mathrm{LiF}-\mathrm{MnO}$ is displayed in a full cell with $\mathrm{Li}_{4} \mathrm{Ti}_{5} \mathrm{O}_{12}$ negative electrode (Supplementary Fig. 5) ${ }^{14}$. LiF-FeO and $\mathrm{LiF}-\mathrm{CoO}$ nanocomposites delivered discharge capacities of 310 and $206 \mathrm{mAh} \mathrm{g}^{-1}$, respectively, demonstrating the general applicability of metal oxides used as positive electrodes when mixed with $\mathrm{LiF}$ (Fig. 2b,c).

\section{Electrochemical response of LiF-MnO nanocomposites}

Pseudocapacitive electrochemical behaviour was observed in $\mathrm{LiF}-\mathrm{MnO}$ nanocomposite from the electrochemical response in the cyclic voltammetry $(\mathrm{CV})$ experiment from $0.075 \mathrm{mV} \mathrm{s}^{-1}$ to $1.00 \mathrm{mV} \mathrm{s}^{-1}$. In the $\mathrm{CV}$ experiment, the redox reaction appeared mainly at $2.5 \mathrm{~V}$ and $3.75 \mathrm{~V}$, which could be ascribed to manganese redox reaction (Fig. 3a). Cathodic and anodic peak currents at each reaction voltage $(2.5 \mathrm{~V}, 3.75 \mathrm{~V})$ tend to increase as the scan rate increases from $0.075 \mathrm{mV} \mathrm{s}^{-1}$ to $1.00 \mathrm{mV} \mathrm{s}^{-1}$. The redox peak currents obey a power-law relationship with the scan rate, which exhibits a nearly linear proportionality of $i_{\mathrm{p}}=a v^{b}(b=\sim 1)$ (Fig. 3b,c), indicating that the electrochemical reaction is primarily surface controlled in contrast to the bulk-diffusion-controlled reaction $(b=0.5)$ generally observed in intercalation-based positive electrodes $^{15}$. The current can be expressed more precisely as the sum of the surface-controlled and diffusion-controlled capacities at a fixed potential ${ }^{16-18}$ :

$$
i(V)=k_{1} v+k_{2} v^{1 / 2}
$$

In equation (1), the $k_{1} v$ and $k_{2} v^{1 / 2}$ terms indicate the surfaceand diffusion-controlled contributions, respectively. The $k_{1}$ and $k_{2}$ values at fixed potentials were determined by the correlation between the scan rates and corresponding current signals according to equation (2) (Supplementary Fig. 6):

$$
i(V) / v^{1 / 2}=k_{1} v^{1 / 2}+k_{2}
$$

Accordingly, the capacitive contribution as a function of the voltage could be obtained using $i(V)=k_{1} v$ at $1 \mathrm{mV} \mathrm{s}^{-1}$ scan rate, as demonstrated in Fig. 3d. The capacity from the surface-controlled reaction accounts for $94 \%$ of the overall capacity by comparing the shaded region with the total area. The remarkable power capability of the $\mathrm{LiF}-\mathrm{MnO}$ nanocomposite ensures the surface-controlled reaction kinetics. The electrode exhibited $110 \mathrm{mAh} \mathrm{g}^{-1}$ discharge capacity even at a current rate of $5,000 \mathrm{~mA} \mathrm{~g}^{-1}$, with a cycle life stable over 100 cycles (Supplementary Fig. 7).

\section{Mn redox reaction aided by the fluorine ions}

The reversible change in the Mn oxidation state was observed during the entire charge and discharge reaction using in situ $\mathrm{X}$-ray absorption near-edge structure analysis (XANES), shown in Fig. 4a,b. Mn oxidation and reduction were evident for both the first and subsequent cycles (Fig. 4c,d). Raman spectroscopy also showed the reversible $\mathrm{Mn}$ redox reaction. The stretching bond strength between $\mathrm{Mn}$ and $\mathrm{O}\left(610-648 \mathrm{~cm}^{-1}\right)$ changed due to variations in the electrostatic attraction force, according to the oxidation and reduction of Mn ions (Supplementary Fig. 8). Similarly, the LiF-FeO nanocomposite also showed that the Fe redox reaction participated in the electrochemical reaction as confirmed by in situ XANES (Supplementary Figs 9 and 10).

As with the reversible oxidation and reduction of $\mathrm{Mn}$, the $\mathrm{Li}$ and F ions in LiF participate as charge carriers and charge neutralizers. Additional experiments excluding any fluorine sources other than $\mathrm{LiF}$, that is, using fluorine-free binder and electrolyte, were carried out in Supplementary Fig. 11, which showed no significant changes in the electrochemical profile, confirming the role of $\mathrm{LiF}$ as the source of F. X-ray photoemission spectroscopy (XPS) of the Li $1 s$ region showed that the lithium signal at $56.0 \mathrm{eV}$ disappears at charge and is recovered at discharge (Fig. 4e). Especially notable is that the extraction of lithium ions during the first charge indicates the decomposition of LiF. In the $\mathrm{F} 1 s$ region shown in Fig. $4 \mathrm{f}$, the binding energy of $685.3 \mathrm{eV}$ from $\mathrm{LiF}$ increased to $686.0 \mathrm{eV}$ after charge and 
a

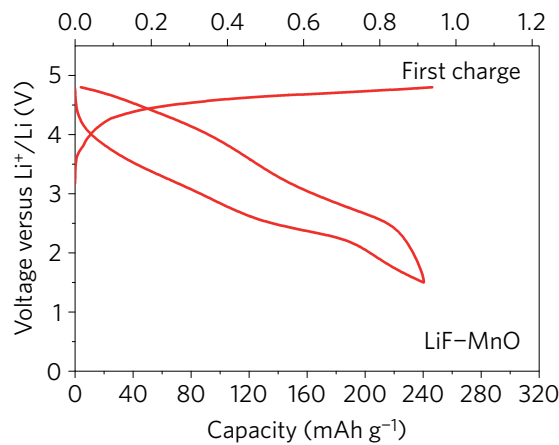

b

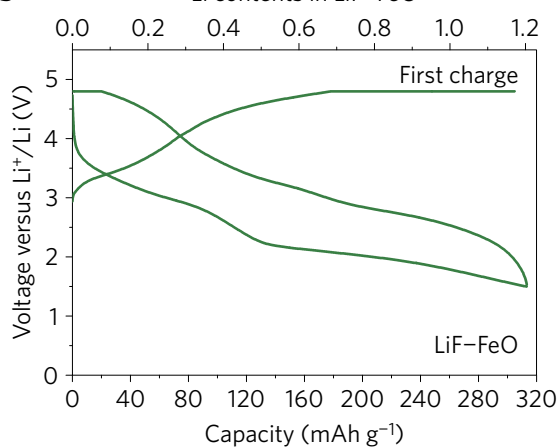

c

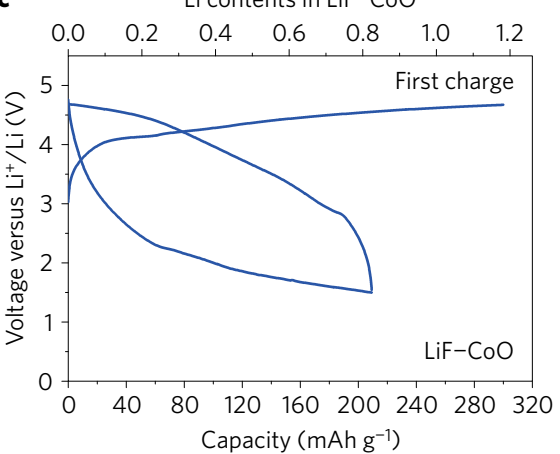

Figure $\mathbf{2}$ | Electrochemical profiles of LiF-MO nanocomposites as a positive electrode. a-c, First discharge and second charge profiles of LiF-MO $(\mathrm{M}=\mathrm{Mn}, \mathrm{Fe}, \mathrm{Co})$ nanocomposites after the initial activating charge protocol as a positive electrode $(1.5-4.8 \mathrm{~V})$ in $1 \mathrm{M} \mathrm{LiPF}_{6}$ in ethyl carbonate/dimethyl carbonate $(E C / D M C ; v / v=1: 1)$ electrolyte at a $20 \mathrm{~mA} \mathrm{~g}^{-1}\left(0.03 \mathrm{~mA} \mathrm{~cm}^{-2}\right)$ constant current rate.

returned to the initial state when discharged. A peak at $686.0 \mathrm{eV}$ comes from the local environment of the $\mathrm{F}^{-}$ion bound to the $\mathrm{Mn}$ ion to satisfy charge neutrality as oxidation occurs.

The electrochemical reaction between the $\mathrm{MnO}$ and $\mathrm{F}$ ions was further investigated using soft X-ray absorption spectroscopy (Fig. 4g). Mn L-edge signals collected from the surface-sensitive total electron yield mode revealed the characteristic peak of $\mathrm{Mn}^{2+}$ $(639.6 \mathrm{eV})$, including a small portion of the $\mathrm{Mn}^{3+}$ signature in the asprepared state, which has been observed in $\mathrm{MnO}$ in many previous works ${ }^{19}$. On charging, the $\mathrm{Mn}^{3+}$ and $\mathrm{Mn}^{4+}$ signals increased at the expense of the $\mathrm{Mn}^{2+}$ signal. The reversible change was observed during the subsequent electrochemical cycling, which indicates that not only $\mathrm{Mn}^{2+} / \mathrm{Mn}^{3+}$ but also $\mathrm{Mn}^{3+} / \mathrm{Mn}^{4+}$ redox reactions are involved in the electrochemical reaction. It was noted that the $\mathrm{Mn}^{3+} / \mathrm{Mn}^{4+}$ reaction mainly participates in the high potential redox reaction $(\sim 3.75 \mathrm{~V})$, while the $\mathrm{Mn}^{2+} / \mathrm{Mn}^{3+}$ redox reaction occurs in the entire voltage range as described in Supplementary Fig. 12.

The F K-edge spectra revealed slight changes in the $685-687.5 \mathrm{eV}$ range (Supplementary Fig. 13). In the charged state, the peak at 685-687.5 eV, which is mainly observed in the manganese fluoride system $\left(\mathrm{MnF}_{2}\right.$ or $\left.\mathrm{MnF}_{3}\right)$, slightly increased ${ }^{20}$. In contrast to the charged state, the peak reversibly decreased in the discharged state. The XPS and X-ray absorption spectroscopy (XAS) results imply that the $\mathrm{Mn}^{2+} / \mathrm{Mn}^{3+}$ and $\mathrm{Mn}^{3+} / \mathrm{Mn}^{4+}$ redox reactions mainly occur on the $\mathrm{MnO}$ surface accompanying $\mathrm{F}^{-}$ion interaction. It is noted that the redox peak at $3.75 \mathrm{~V}$ in the $\mathrm{CV}$ experiment (Fig. 3a) accompanies the $\mathrm{Mn}^{3+} / \mathrm{Mn}^{4+}$-related redox reaction as shown in Supplementary Fig. 12. Additionally, the pseudocapacitive behaviour may be due to the surface redox reaction of $\mathrm{MnO}$ with the $\mathrm{F}^{-}$ion from LiF. However, the current response does not establish the reaction mechanism; therefore, we further investigated the reaction mechanism using $\mathrm{TEM}^{21}$.

\section{Surface-concentrated chemical and structural changes}

The chemical distribution information for the charged and discharged states was obtained using scanning TEM coupled with electron energy-loss spectroscopy (STEM-EELS) spectrum images (Fig. 5a,b). We minimized the electron beam dose with optimized conditions (Supplementary Fig. 14). We observed both Mn and F signals from the charged and discharged states and confirmed that the $\mathrm{F}^{-}$ion distribution was not homogeneous but preferentially occurred on the $\mathrm{MnO}$ surface. It was generally observed that the fluorine distribution was mainly concentrated on the surface of the particle with approximately $2-5 \mathrm{~nm}$ thickness regardless of the charge and discharge state for most of the particles, which indicates that the electrochemical reaction with fluorine occurs relatively homogeneously on the surface of the electrode material (Supplementary Fig. 15). This trend is different from that for the iron-based oxyfluoride phase, which has a core/shell structure with an O-rich shell and a F-rich core ${ }^{22}$. F coexists with Mn in the surface region in the charged state; however, they tend to exist separately in the discharged state. This result is possibly due to the reversible interaction between $\mathrm{Mn}$ and $\mathrm{F}$ during electrochemical cycling. Therefore, we compared the valence of $\mathrm{Mn}$ in the F-rich shell and F-less core region to clarify the reversible interaction between $\mathrm{Mn}$ and $\mathrm{F}$ (Fig. $5 \mathrm{c}, \mathrm{d}$ ). A significant change in the $\mathrm{Mn} \mathrm{L}_{3}$ peak energy was observed in the $\mathrm{Mn} \mathrm{L}_{2,3}$ EELS spectra in the charged state. The $\mathrm{Mn} \mathrm{L}_{3}$ peak observed for the F-rich shell $(641.0 \mathrm{eV})$ has a higher value than $639.8 \mathrm{eV}$ for the F-less core; however, the peak energies from both the shell and core regions were similar in the discharged state. The non-uniform $\mathrm{Mn}$ valence distribution across the single particle in the charged state supports the surface-concentrated Mn redox reaction (Supplementary Fig. 16). This finding indicates that the $\mathrm{F}^{-}$ion incorporation accompanying the redox reaction of $\mathrm{Mn}$ ions mainly occurs on the $\mathrm{MnO}$ surface.

On charge, significant local structure evolution was observed including attenuation of the rock salt signature and a decrease of the bond distance from in situ extended X-ray absorption finestructure (EXAFS) analysis (Fig. 5e). X-ray diffraction analyses of the electrode materials do not provide insight into the nature of charged/discharged product due to the loss of long-range ordering during the first charge (Supplementary Fig. 17). Meanwhile, EXAFS showed evident local structure changes during electrochemical cycling. The signal at longer distances ( $4-6 \AA$ ) gradually decreased at initial charging, indicating a reduction in crystallinity. In addition, the electrode undergoes a decrease of bond distances at the first and second neighbour. The shortened bond distance of the first neighbour at charge is similar to manganese compounds, which have a higher oxidation state than $\mathrm{Mn}^{2+}$ (Supplementary Fig. 18). At the end of charge, the electrode showed completely different local structure from the pristine state with the attenuated second neighbour ${ }^{23-26}$. This amorphization and attenuation of the rock salt phase signature were similarly observed in the iron oxyfluoride system during the phase transformation from rock salt to the F-rich rutile phase when fluorine interacts with the rock salt phase through the reconversion reaction ${ }^{26}$. However, the charged state of the $\mathrm{LiF}-\mathrm{MnO}$ nanocomposite exhibits a distinct local structure from the rutile-type manganese fluoride phase and retained the cubic local structure similar to the $\mathrm{MnO}_{x}$ phase (Supplementary Fig. 18).

The reversible recovery of the local structure as the rock salt phase was demonstrated after the discharge. The electrode at the end of discharge exhibits similar features to those of the pristine electrode in the local range of 1-6 $\mathrm{A}$. The first and second neighbour recovered similarly to those in the as-prepared $\mathrm{MnO}$, and the peaks in the 4-6 $\AA$ range reemerged, implying the reformation of the MnO-like crystal structure. The local structure of the 

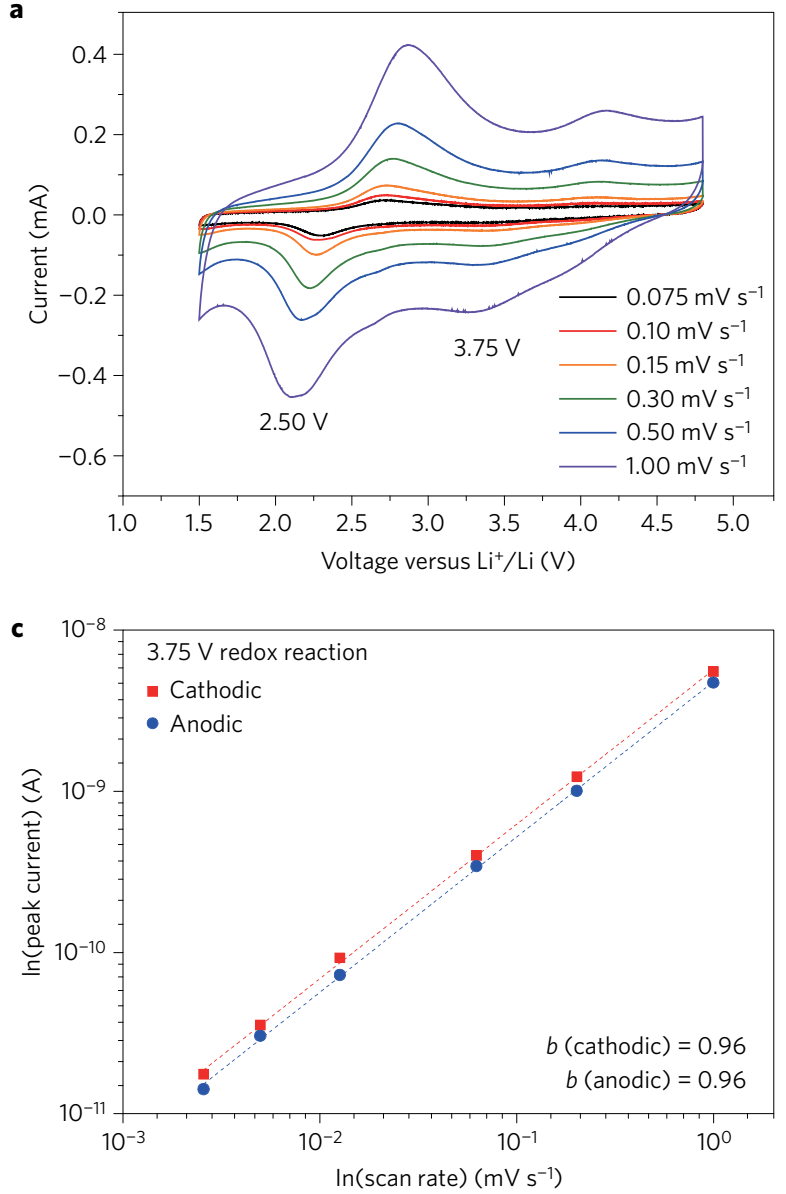

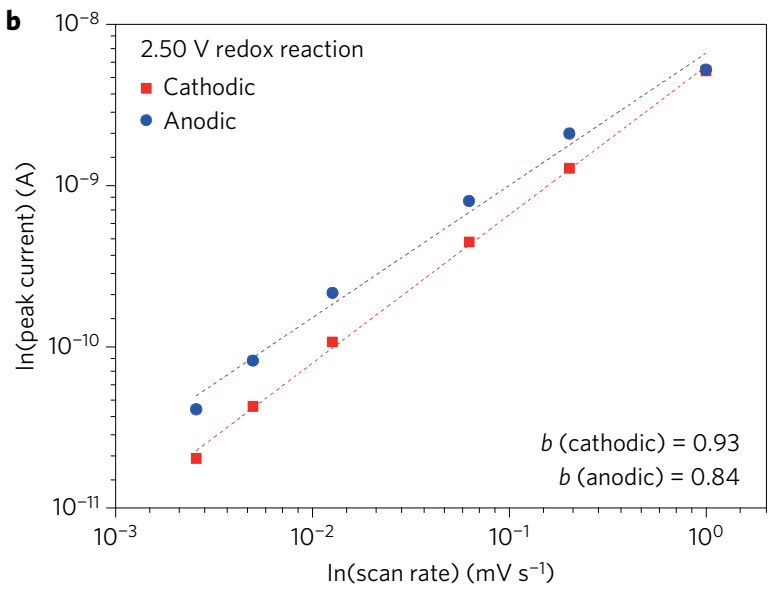

d

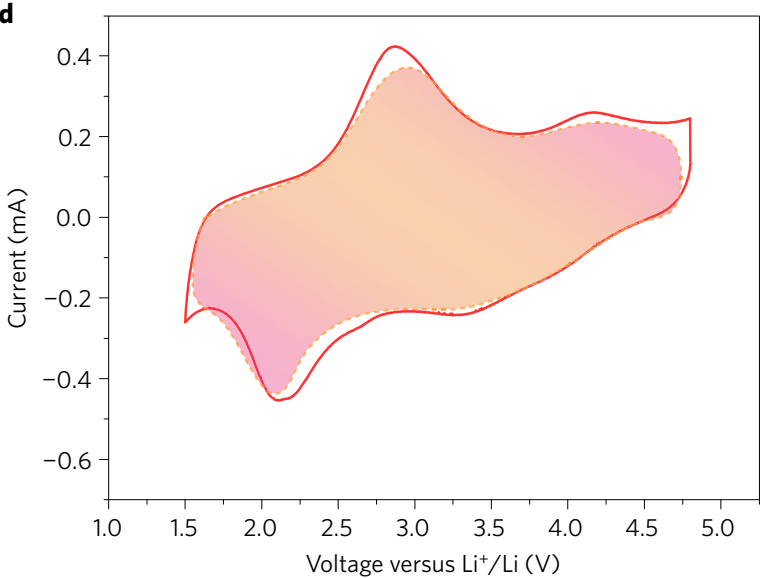

Figure 3 | Electrochemical response of the LiF-MnO nanocomposite. a, Cyclic voltammetry at various scan rates from 0.075 to $1.00 \mathrm{mV} \mathrm{s}{ }^{-1}$ after the first charge to $4.8 \mathrm{~V}$ at a constant current density $\left(20 \mathrm{~mA} \mathrm{~g}^{-1}\right)$. b,c, Log-scale plot of peak current $(2.5 \mathrm{~V}, 3.75 \mathrm{~V}$ redox reaction) dependence on the scan rate $\left(i_{p}\right.$ versus $v$ ). The dotted lines indicate the linear fit of cathodic and anodic current as scan rate. The $b$ value indicates the slope of the linear fit curve. $R^{2}$ and standard error of each slope are shown in the Methods. d, Current response with voltage at $1 \mathrm{mV} \mathrm{s}^{-1}$ scan rate. The overall current signal (solid line) was obtained from the cyclic voltammetry experiment. Capacitive current (shaded region with dotted line) was calculated using $i(V)=k_{1} v$.

discharged state was well matched with the standard rock salt phase $\mathrm{MnO}$ model, suggesting that the structural transition is reversible during electrochemical cycling (Fig. 5e and Supplementary Fig. 19). This reversible behaviour was observed continuously during the subsequent electrochemical cycling.

A reversible local structure change was observed on the $\mathrm{MnO}$ surface in high-resolution high-angle annular dark-field images (Fig. 5f,g). The atomic-resolution image of the charged state reveals the different atomic structure in the F-rich shell region compared with that in the F-less core region. The fast Fourier transformation (FFT) image of the core region reveals a periodic pattern projected to the [011] zone axis of $F m \overline{3} m \mathrm{MnO}$, which indicates that the core region is well maintained as the $\mathrm{MnO}$ phase. Meanwhile, an additional pattern with a large $d$-spacing $(5.02 \AA)$ was observed in the shell region. The local structure is analogous to the $I 4_{1} /$ amd space group $\mathrm{Mn}_{3} \mathrm{O}_{4}$ structure considering the crystallographic orientation [011] zone axis of $F m \overline{3} m \mathrm{MnO}$, which is coherent with the [010] zone axis of the $\mathrm{I}_{1} /$ amd space group $\mathrm{Mn}_{3} \mathrm{O}_{4}$ (Supplementary Fig. 20). However, the surface structure was defectively formed, including a vacancy on the $\mathrm{Mn}$ tetrahedral site of $\mathrm{Mn}_{3} \mathrm{O}_{4}$ (Fig. 3f). This result may be due to the instability of $\mathrm{Mn}^{3+}$ or $\mathrm{Mn}^{4+}$ ions in tetrahedral sites compared with octahedral sites considering the oxidized environment of the surface region by $\mathrm{F}^{-}$ion incorpora$\operatorname{tion}^{27,28}$. This face-centred cubic to body-centred tetragonal phase transition is known for the diffusionless transformation ${ }^{29}$, which indicates that the transformation kinetics could be sufficiently fast to accommodate the high current density during the electrochemical cycling (Supplementary Fig. 7). Given the coherent distribution of the structure transformed region and $\mathrm{F}^{-}$ions (Fig. 5a and Supplementary Fig. 21), the surface local structure of the charged state is expected to be a defective $\mathrm{Mn}-\mathrm{O}-\mathrm{F}$-type spinel-like phase. In contrast to the charged state, the discharged electrode exhibited a uniform structure even in the shell region as the $\mathrm{MnO}$ rock salt phase (Fig. 5g,h and Supplementary Fig. 22).

This reversible structure evolution is identical to the results from in situ EXAFS. As the thickness of the shell region is approximately 2-4 nm (Fig. 5a,f), if we assume the particle size of $\mathrm{MnO}$ as $10 \mathrm{~nm}$, the surface layer occupies $78.4 \%$ of the $\mathrm{MnO}$ single nanoparticle (Supplementary Fig. 23). Therefore, the local structure change from the in situ EXAFS can be originated from the structure evolution on the $\mathrm{MnO}$ surface considering the particle size level.

\section{Particle size effect on electrochemistry}

Simple numerical calculations, assuming the involvement of the $\mathrm{Mn}$ redox reaction up to $2 \mathrm{~nm}$ from the $\mathrm{MnO}$ surface, confirm the feasibility of a large capacity $\left(>236.37 \mathrm{mAh} \mathrm{g}^{-1}\right)$ for submicrometrescale particles $(\sim 10 \mathrm{~nm})$. On the basis of the simplified model from the first-principles calculations, the capacity could be numerically predicted as a function of the particle size of $\mathrm{MnO}$ (Supplementary Figs 24-27 and Supplementary Text). In particular, the large capacity from the surface reaction was feasible because of the twoelectron reaction of the $\mathrm{Mn}$ ion $\left(\mathrm{Mn}^{2+} / \mathrm{Mn}^{3+}\right.$ and $\left.\mathrm{Mn}^{3+} / \mathrm{Mn}^{4+}\right)$ on 

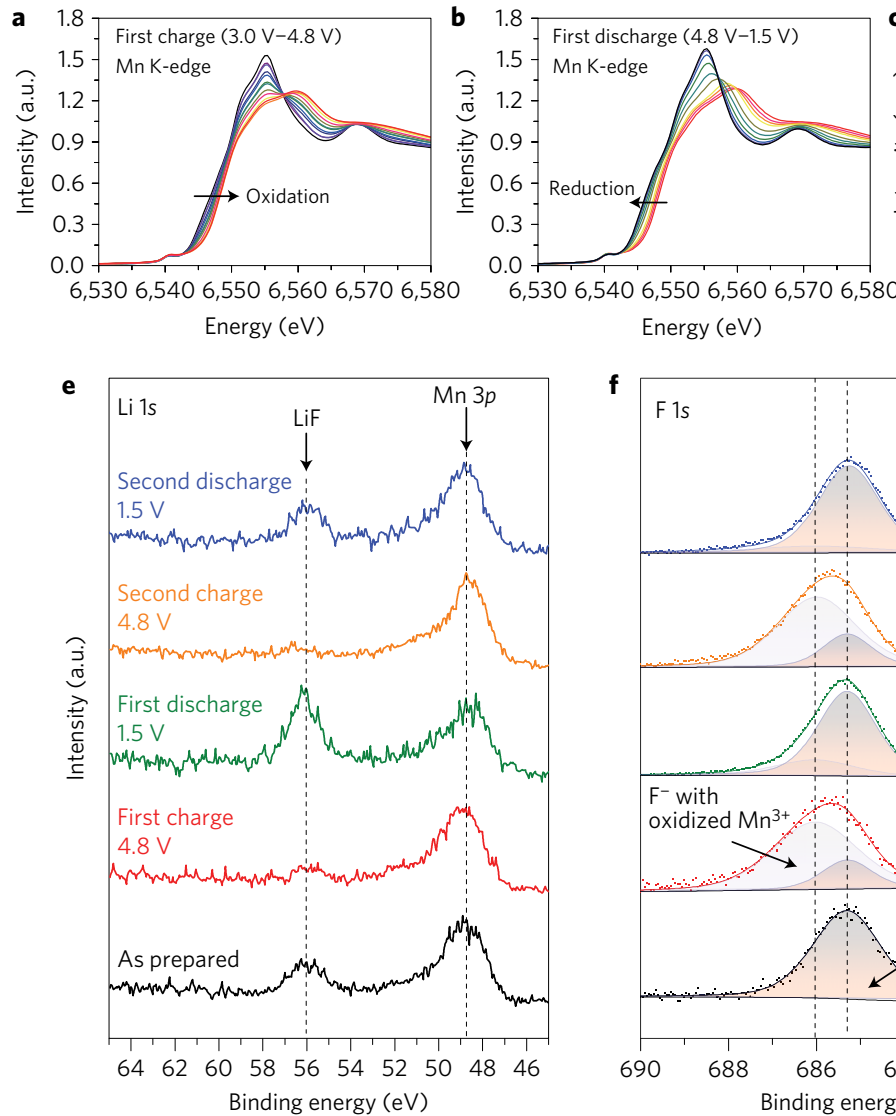
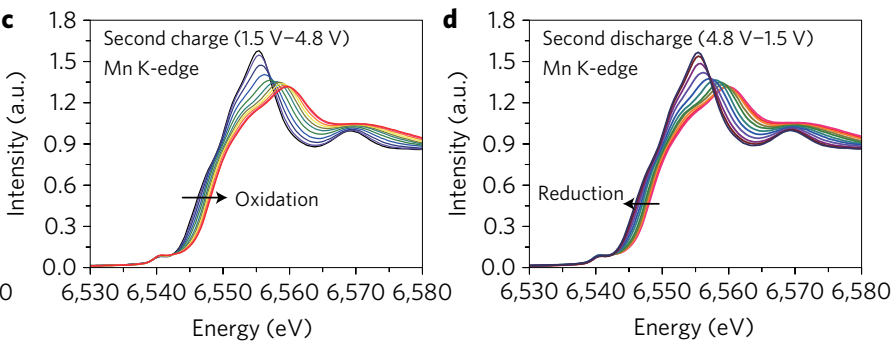
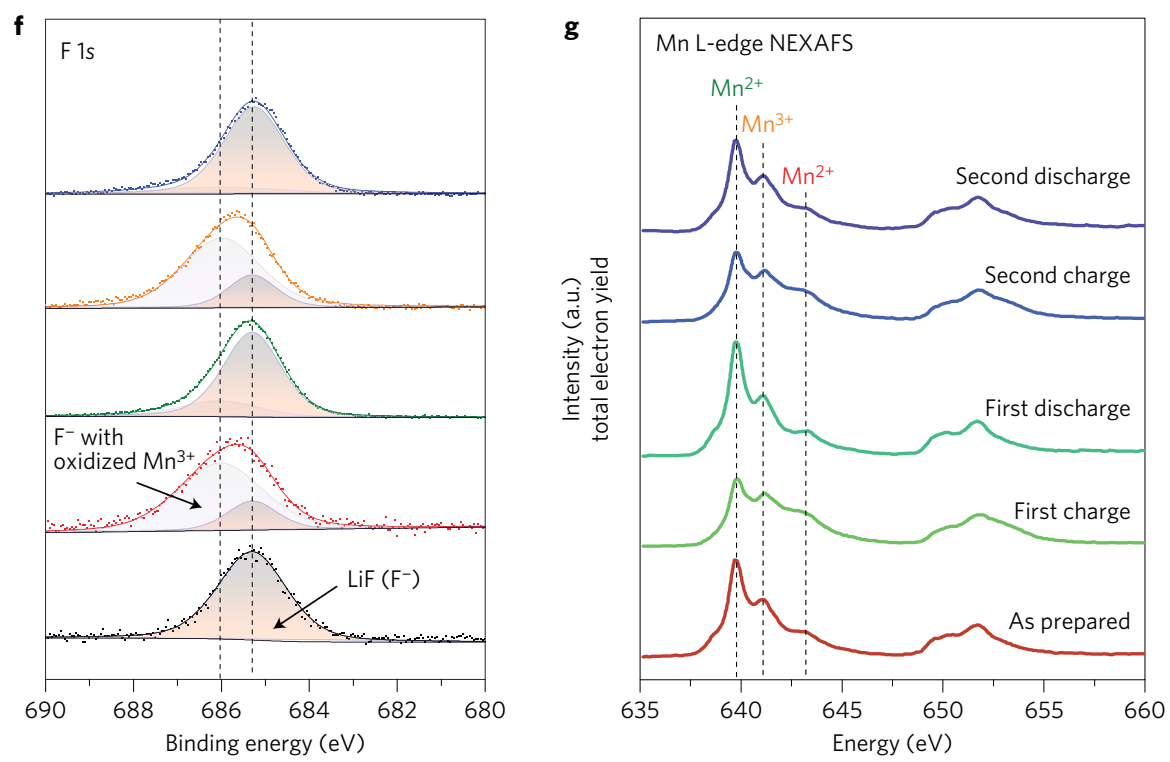

Figure 4 | Mn redox reaction and fluorine ion contribution. a-d, In situ XANES spectra of the Mn K-edge for the first two cycles in the 1.5-4.8 $\mathrm{V}$ range. e, Ex situ XPS spectra of Li 1s, including the range of Mn 3p binding energy. The peaks were normalized to the Mn $3 p$ peak to compare Li contents in the electrode. f, Fluorine 1s photoelectrons were gathered from the same position as Li 1s. To remove uncertainty from the analysis, the fluorine sources except $\mathrm{LiF}$ were eliminated using polyacrylonitrile (PAN) as a binder and ethyl carbonate/dimethyl carbonate $(\mathrm{EC} / \mathrm{DMC} ; \mathrm{V} / \mathrm{v}=1: 1)$ in a $1 \mathrm{M} \mathrm{LiClO} 4$ electrolyte $\left(\mathrm{LiClO}_{4}\right.$ should be used carefully due to its explosive nature). $\mathbf{g}, \mathrm{Mn}$ L-edge NEXAFS spectra of the LiF-MnO nanocomposite from total electron yield mode with different electrochemical states. The vertical dashed lines indicate the edge position of the manganese ion with different oxidation states.

the $\mathrm{MnO}$ surface (Supplementary Fig. 26). In the simulation, we observed that the capacity can be dramatically increased as the particle size decreases below $10 \mathrm{~nm}$ because of the larger surface area of the small-particle electrodes compared with large-particle ones. Our experimental results for the capacity $\left(\sim 240 \mathrm{mAh}^{-1}\right)$ with $\sim 10$-nm particle sizes match with the capacity predicted on the basis of the numerical simulations (Supplementary Fig. 27).

The particle size effect of the metal compound on the electrochemical activity in the nanocomposite cathode material was verified with systematic control of the particle size and shape of manganese oxide (Fig. 6a and Supplementary Fig. 28). $\mathrm{Mn}_{3} \mathrm{O}_{4}$ was selected as the metal oxide compound to compare the electrochemical activity as a function of particle size. A $\mathrm{LiF}-\mathrm{C} / \mathrm{Mn}_{3} \mathrm{O}_{4}$ nanocomposite electrode was fabricated by making a mixture with $\mathrm{LiF}-\mathrm{C}$ and as-synthesized $\mathrm{Mn}_{3} \mathrm{O}_{4}$ nanoparticles without additional high-energy ball milling ${ }^{30}$. $\mathrm{LiF}$ and $\mathrm{Mn}_{3} \mathrm{O}_{4}$ were well mixed in the carbon matrix without a change in particle size or morphology (Fig. 6b and Supplementary Fig. 29). The $\mathrm{LiF}-\mathrm{C} / \mathrm{Mn}_{3} \mathrm{O}_{4}$ mixture exhibited electrochemical activity as a cathode material with a $3.5-\mathrm{V}$ average voltage redox reaction after the first charge (Supplementary Fig. 30). The capacity increased from 96 to $159 \mathrm{mAh} \mathrm{g}^{-1}$ as the particle size decreased from 20 to $6 \mathrm{~nm}$ (Fig. 6c). This result indicates that the particle size of $\mathrm{Mn}_{3} \mathrm{O}_{4}$ directly affects the capacity of the $\mathrm{LiF}-\mathrm{C} / \mathrm{Mn}_{3} \mathrm{O}_{4}$ nanocomposite cathode and implies that the particle size of metal compounds can be a feasible tool for tuning the capacity in the nanocomposite cathode material system.

\section{Reaction mechanism}

Therefore, the reaction mechanism of the $\mathrm{LiF}-\mathrm{MnO}$ nanocomposite can be called a 'surface conversion reaction', which is described as follows:

$$
\mathrm{LiF}+\mathrm{Mn}^{2+} \mathrm{O} \rightleftarrows \mathrm{Li}^{+}+\mathrm{e}^{-}+\mathrm{Mn}^{3+} \mathrm{O}-\mathrm{F}
$$

The surface conversion reaction $(\mathrm{MX}+\mathrm{LiA} \leftrightarrow \mathrm{MX}-\mathrm{A}+\mathrm{Li})$ can take advantage of the high-valence redox reaction of transition metals because the capacity of the LiF-MO nanocomposite is fully utilized based on the $\mathrm{M}^{2+} / \mathrm{M}^{3+}$ or $\mathrm{M}^{3+} / \mathrm{M}^{4+}$ redox reaction. The transition metal is always housed by the anion (by the $\mathrm{X}$ anion in the discharged state or by $\mathrm{X}$ and $\mathrm{A}$ anions in the charged state) in this reaction and can thus maintain the high-valence state offered by the anions and the high-voltage operation of the electrode. LiF functions not only as a Li source for cathode materials but also as an anion source for charge compensation when the oxidation and reduction of a transition metal ion occur. LiF can be regarded as a stabilizer of the oxidized $\mathrm{Mn}^{3+}$ (or $\mathrm{Mn}^{4+}$ ) ion by providing $\mathrm{F}^{-}$ions from the $\mathrm{MnO}$ perspective. In addition, the $\mathrm{MnO}$ can be regarded as an assistant that promotes the decomposition of $\mathrm{LiF}$. $\mathrm{LiF}$ is difficult to decompose electrochemically by itself because it is a stable ionic compound with a substantially large formation energy corresponding to $6.10 \mathrm{~V}$ versus $\mathrm{Li}^{+} / \mathrm{Li}$ (Supplementary Table 1). It is expected that the electrochemical activity of the $\mathrm{LiF}-\mathrm{MnO}$ nanocomposite originates from a series of reactions in which a slightly oxidized $\mathrm{Mn}^{(2+\delta)+}$ ion is formed by the extraction of an 


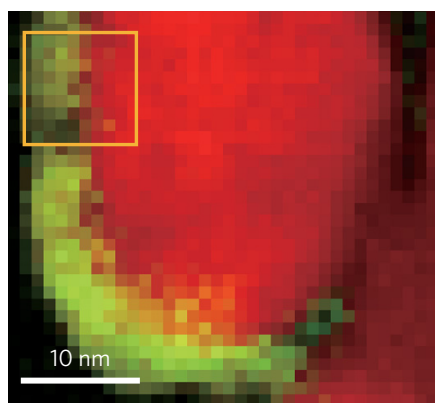

b

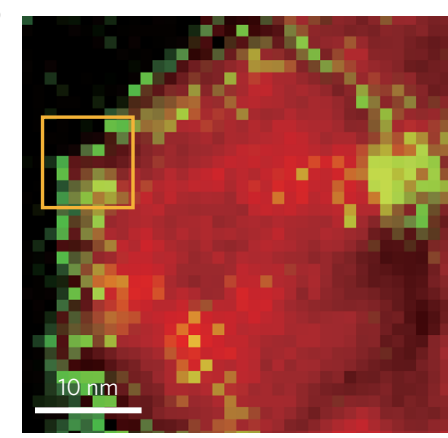

c

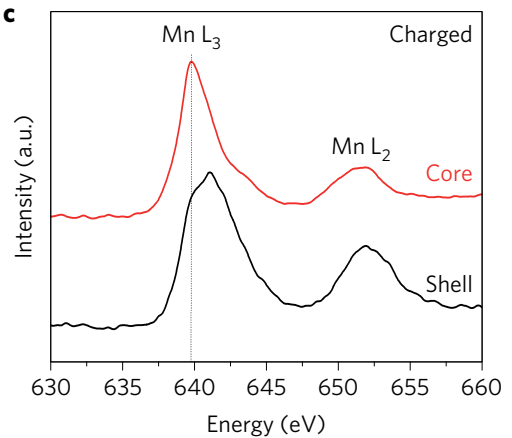

d

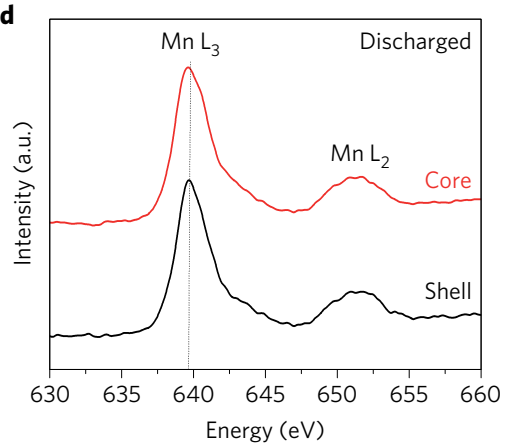

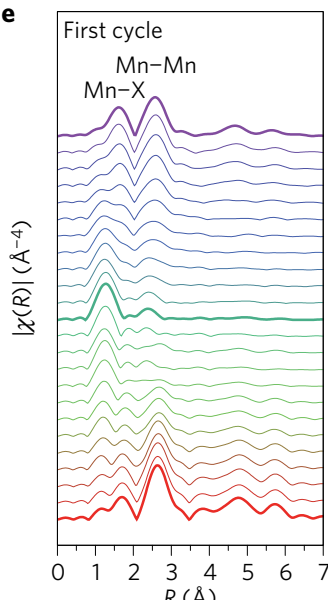

$R(\AA)$
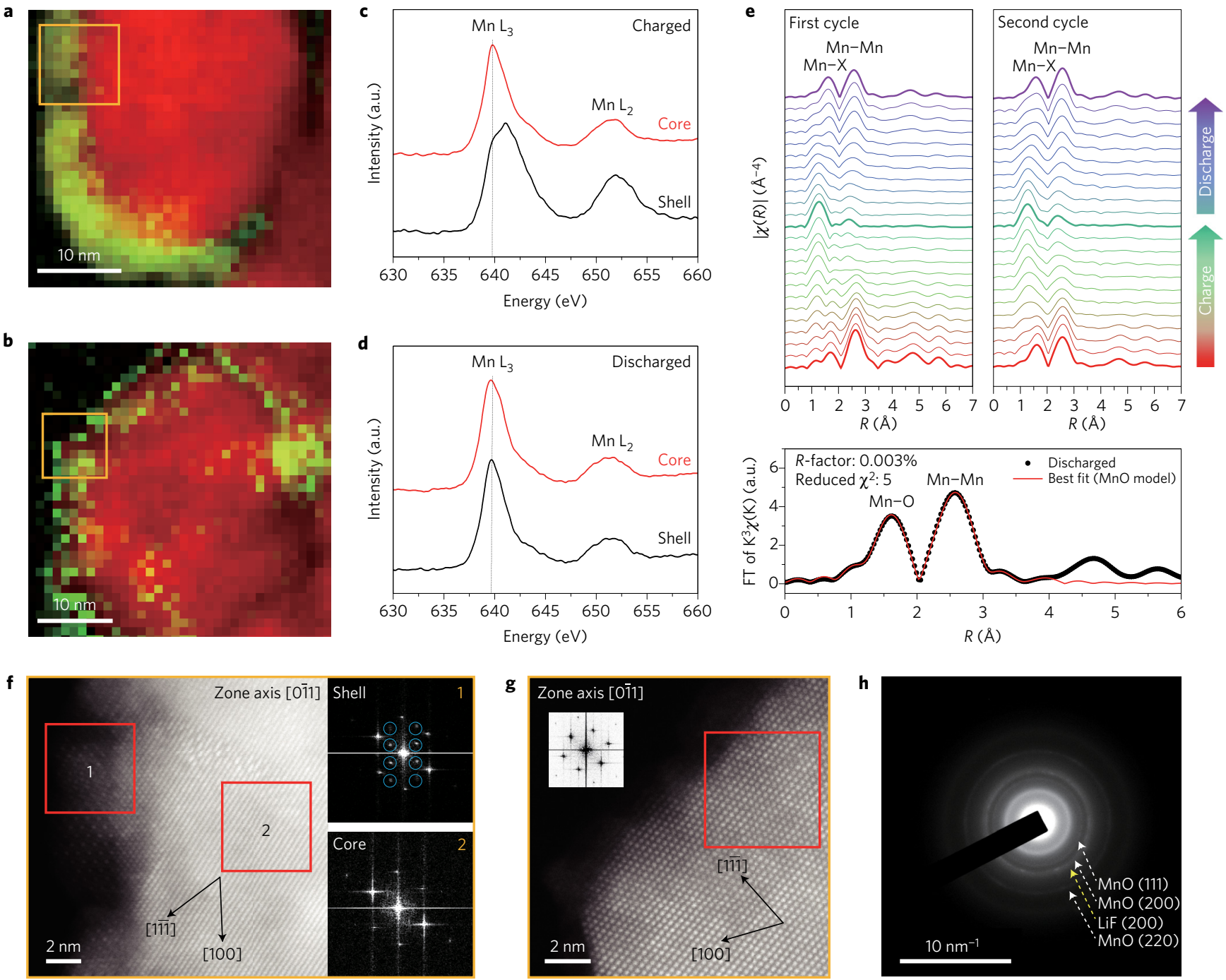

Figure $\mathbf{5}$ | Surface-concentrated $\mathbf{F}^{-}$ion interaction including structural evolution. $\mathbf{a}, \mathbf{b}$, STEM-EELS images of the LiF-MnO nanocomposite in the charged (a) and discharged (b) states for the elemental distribution of $F$ (green) and $\mathrm{Mn}$ (red). c,d, EELS spectra of Mn $\mathrm{L}_{2,3}$-edges recorded from the shell and core regions of the charged (c) and discharged (d) states. e, In situ EXAFS spectra during two cycles in the 1.5-4.8 V range. The first charge is operated in the 3.0-4.8 V range. The EXAFS data and the best-fit model are plotted for the discharged electrode. $\mathbf{f}, \mathbf{g}$, High-resolution high-angle annular dark-field images of the charged state $(4.8 \mathrm{~V})(\mathbf{f})$ and the discharged state $(1.5 \mathrm{~V})(\mathbf{g})$ for the areas indicated by the yellow boxes in $\mathbf{a}$ and $\mathbf{b}$, respectively. The FFT pattern was obtained for the areas indicated by the red boxes. The blue circles indicate an additional FFT pattern compared with the core region. $\mathbf{h}$, Diffraction pattern of an electrode discharged to $1.5 \mathrm{~V}$.

electron during the initial charge, followed by the decomposition of $\mathrm{LiF}$. Therefore, $\mathrm{LiF}$ can donate the $\mathrm{F}^{-}$ion to stabilize the oxidized $\mathrm{Mn}$ ion of $\mathrm{Mn}^{3+}$ or $\mathrm{Mn}^{4+}$ when it decomposes. This process can occur only when $\mathrm{LiF}$ and $\mathrm{MnO}$ compounds are intimately well mixed on the nanoscale.

We found that the reaction mechanisms of $\mathrm{LiF}-\mathrm{MO}(\mathrm{M}=\mathrm{Fe}, \mathrm{Co})$ nanocomposites are different compared with the case of $\mathrm{LiF}-\mathrm{MnO}$. (See Supplementary Information for details.) Both LiF-MO $(\mathrm{M}=\mathrm{Fe}, \mathrm{Co})$ nanocomposites showed electrochemical activity based on the $\mathrm{M}^{2+} / \mathrm{M}^{3+}$ redox reaction, which was enabled by the interaction with fluorine ions. However, the specific fluorine ion behaviour was slightly different depending on the transition metal compound. In the $\mathrm{LiF}-\mathrm{FeO}$ case, fluorine ions were better incorporated into the host structure due to the thermodynamic stability of the FeOF compound. As a result, this formulation showed remarkably similar electrochemical properties and local structure changes to those of $\mathrm{Li}_{x} \mathrm{FeOF}$ (Supplementary Figs 31-33 and Supplementary Text) during subsequent discharge and charge ${ }^{25,26}$, similar to our previously reported case of $\mathrm{LiF}-\mathrm{FeF}_{2}$, which can be termed a 'host formation reaction's. In the case of $\mathrm{LiF}-\mathrm{CoO}$, although the trend of change in oxidation state and local structure is similar to the case of $\mathrm{LiF}-\mathrm{MnO}$, the changes are relatively small compared with the reversible capacity (Supplementary Figs 34 and 35). It implies that $\mathrm{LiF}-\mathrm{CoO}$ could have a different charge compensation mechanism including not only Co redox reaction but also other reaction mechanisms, which needs to be revealed by in-depth study as future work. This difference in reaction processes can be determined by whether the fluorinated phase of the initial metal compound occurs. Note that the iron oxyfluoride phase exists, while the manganese or cobalt oxyfluoride phase has not been reported ${ }^{31}$. The interaction behaviour of the $\mathrm{F}^{-}$ion with metal compounds and the thermodynamic stability of the charged form seem to determine the overall reaction mechanism.

\section{Conclusions}

An understanding of this unusual electrochemical reaction behaviour can offer new design criteria for positive electrode materials in lithium-ion batteries. For positive electrode material design, we 

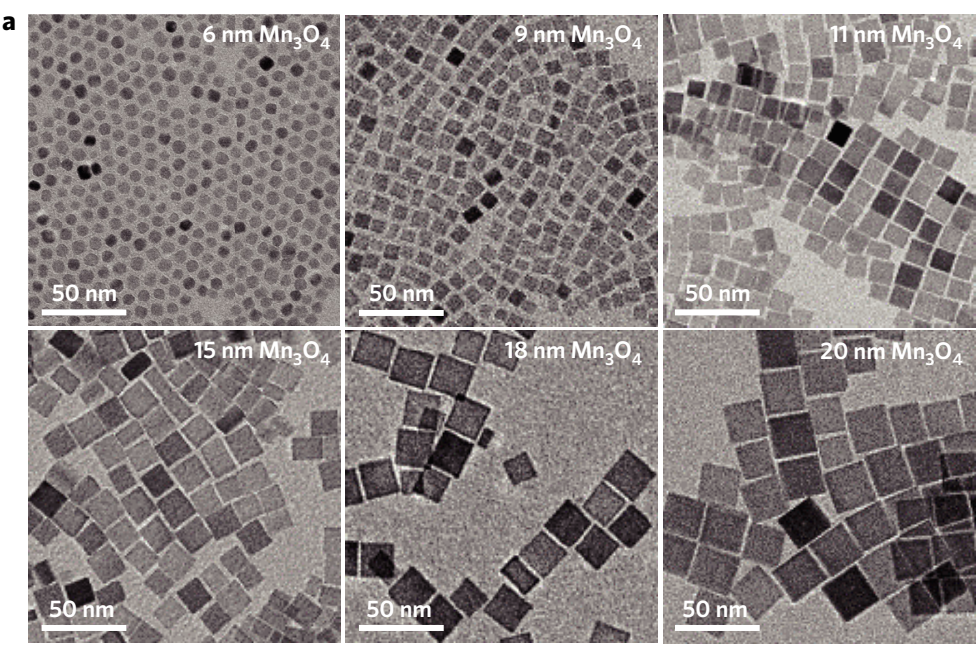

b

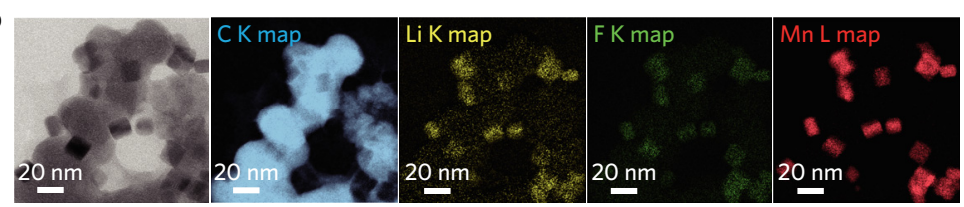

c Li contents in $\mathrm{LiF}-\mathrm{Mn}_{3} \mathrm{O}_{4}$ mixture

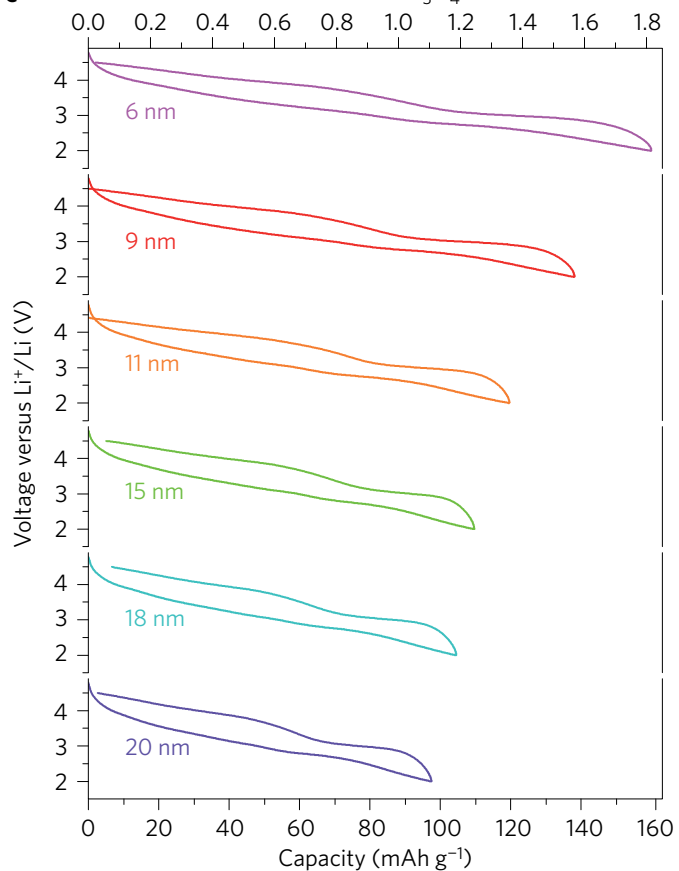

Figure 6 | Capacity-tunable LiF-C/ $\mathrm{Mn}_{3} \mathrm{O}_{4}$ nanocomposite cathode materials. a, TEM images of $\mathrm{Mn}_{3} \mathrm{O}_{4}$ nanoparticles. b, TEM and energy-filtered TEM images of $\mathrm{LiF}-\mathrm{C} / \mathrm{Mn}_{3} \mathrm{O}_{4}(15 \mathrm{~nm})$ mixture electrode in the as-prepared state. $\mathbf{c}$, Electrochemical profile of $\mathrm{LiF}-\mathrm{C} / \mathrm{Mn}_{3} \mathrm{O}_{4}$ nanocomposite with various particle sizes after the first charge.

believe that numerous combinations of transition metal compounds and lithium compounds (other than $\mathrm{LiF}$ ) are possible, and a variety of redox nanocomposite positive electrodes could be developed with tuning of their electrochemical performances. As long as the transition metal compounds contain a valid redox-active component, anion participation may activate the lithium storage capability regardless of the crystal structure. Also, as the lithium compounds are readily decomposable in the electrochemical cell, it can supply lithium ions in the lithium-ion cells. Moreover, the choice of anions in lithium compounds can be used as an additional rudder to tune the electrochemical properties of the composite electrode.

\section{Methods}

Synthesis of MnO/C nanocomposite. $\mathrm{MnO} / \mathrm{C}$ nanocomposites were synthesized by planetary ball milling (Pulverisette 5; Fritsch). As-received $\mathrm{MnO}$ (99\%; Sigma-Aldrich) and fluorine-free graphite (Alfa Aesar) were used without further purification. $\mathrm{MnO}$ and graphite were inserted into the same container $(\mathrm{MnO} / \mathrm{C}$ : $1 / 0.2$, w/w) with an argon atmosphere in the glove box. The powders were mixed in the container at 400 r.p.m. for $48 \mathrm{~h}$ without rest time.

Synthesis of LiF-MO nanocomposite. To synthesize the LiF-MO (M = Mn, Fe, Co) nanocomposite, as-received LiF (99.5\%; Sigma-Aldrich) and MO ( $\mathrm{M}=\mathrm{Mn}, \mathrm{Fe}, \mathrm{Co}$ ) (99\%; Sigma-Aldrich) were used without further purification. Composite material was prepared by planetary milling (Pulverisette 5; Fritsch). $\mathrm{LiF}, \mathrm{MO}(\mathrm{M}=\mathrm{Mn}, \mathrm{Fe}, \mathrm{Co})$, and graphite (20 wt $\%$ of the LiF-MO composite) were inserted into the same container $(\mathrm{Li} / \mathrm{M}=1.2 / 1, \mathrm{~m} / \mathrm{m})$, and the container was sealed in an argon-filled glove box to minimize air exposure. The inserted powders were homogeneously mixed in the container at 400 r.p.m. for $48 \mathrm{~h}$ without rest time to obtain a finely dispersed composite. The synthesized LiF-MO nanocomposite was characterized by X-ray diffraction (XRD) using a D2 PHASER (Bruker) equipped with Cu K $\alpha$ radiation $(\lambda=1.54178 \AA$ ) at a scanning speed of $0.5^{\circ} \mathrm{min}^{-1}$ in a $2 \theta$ range of $30^{\circ}-70^{\circ}$. Fourier transform infrared and Raman spectroscopy were performed to check the formation of impurities in the amorphous phase.

Synthesis of $\mathrm{Mn}_{3} \mathrm{O}_{4}$ nanocrystals. For the synthesis of $\mathrm{Mn}_{3} \mathrm{O}_{4}$ nanocrystals with edge length of $6 \mathrm{~nm}, 4.9 \mathrm{~g}$ manganese (II) acetate tetrahydrate (Aldrich), $53.4 \mathrm{~g}$ oleylamine (Acros) and $5.7 \mathrm{~g}$ oleic acid (Aldrich) were dissolved in $300 \mathrm{ml}$ of 1-octanol (Aldrich). The mixture were stirred at room temperature over $3 \mathrm{~h}$ in a three-necked $1,000-\mathrm{ml}$ flask. The reaction mixture was slowly heated to $120^{\circ} \mathrm{C}$ in air under magnetic stirring, and before it reached $120^{\circ} \mathrm{C}, 3.8 \mathrm{ml}$ of deionized water was rapidly injected into the reaction solution. The solution was aged at $120^{\circ} \mathrm{C}$ in air for $2 \mathrm{~h}$. The solution was then cooled to room temperature and washed with acetone and ethanol. The $\mathrm{Mn}_{3} \mathrm{O}_{4}$ nanocrystals were retrieved by centrifugation. The ligand exchange procedure using hexamethonium hydroxide solution (HMH; Aldrich) was as follows. First, $0.3 \mathrm{~g}$ as-prepared $\mathrm{Mn}_{3} \mathrm{O}_{4}$ nanocrystals were dissolved in $10 \mathrm{ml}$ of tetrahydrofuran solution. Second, $\sim 1 \mathrm{ml}$ of $\mathrm{HMH}$ and $\sim 0.5 \mathrm{ml}$ of deionized water were added to the solution. Then, the nanocrystals were precipitated with ethanol by centrifugation.

For the synthesis of $\mathrm{Mn}_{3} \mathrm{O}_{4}$ nanocrystals with edge length of $9 \mathrm{~nm}, 4.9 \mathrm{~g}$ manganese (II) acetate tetrahydrate (Aldrich), $53.4 \mathrm{~g}$ oleylamine (Acros) and $2.8 \mathrm{~g}$ oleic acid (Aldrich) were dissolved in $300 \mathrm{ml}$ of 1-octanol (Aldrich). The mixture was stirred at room temperature over $3 \mathrm{~h}$ in a three-necked 1,000-ml flask. The reaction mixture was slowly heated to $120^{\circ} \mathrm{C}$ in air under magnetic stirring, and before it reached $120^{\circ} \mathrm{C}, 3.8 \mathrm{ml}$ of deionized water was rapidly injected into the reaction solution. The solution was aged at $120^{\circ} \mathrm{C}$ in air for $2 \mathrm{~h}$. The solution was then cooled to room temperature and washed with acetone and ethanol. The $\mathrm{Mn}_{3} \mathrm{O}_{4}$ nanocrystals were retrieved by centrifugation. The ligand exchange procedure using $\mathrm{HMH}$ was the same as that described above for the 6-nm-sized nanoparticles.

For the synthesis of $\mathrm{Mn}_{3} \mathrm{O}_{4}$ nanocrystals with edge length of $11 \mathrm{~nm}, 6.4 \mathrm{~g}$ manganese (II) acetate tetrahydrate (Aldrich), $17.4 \mathrm{~g}$ oleylamine (Acros) and $3.7 \mathrm{~g}$ oleic acid (Aldrich) were dissolved in $390 \mathrm{ml}$ of xylene (Aldrich). The mixture was stirred at room temperature for $3 \mathrm{~h}$ in a three-necked 1,000-ml flask. The reaction mixture was slowly heated to $100^{\circ} \mathrm{C}$ in air under magnetic stirring, and after it reached $100^{\circ} \mathrm{C}, 26 \mathrm{ml}$ of deionized water was rapidly injected into the reaction solution. The solution was aged at $90^{\circ} \mathrm{C}$ in air for $1.5 \mathrm{~h}$. The solution was then cooled to room temperature and washed with ethanol. The $\mathrm{Mn}_{3} \mathrm{O}_{4}$ nanocrystals were retrieved by centrifugation. The ligand exchange procedure was performed using nitrosonium tetrafluoroborate $\left(\mathrm{NOBF}_{4}\right)^{32} ; 0.035 \mathrm{~g} \mathrm{NOBF}_{4}$ was dissolved in $1.5 \mathrm{ml}$ of dimethylformamide in an Ar atmosphere. This $1.5 \mathrm{ml}$ of $\mathrm{NOBF}_{4}$ solution was then added to the $10 \mathrm{ml}$ of tetrahydrofuran solution of as-prepared nanocrystals. After the ligand exchange process, the nanocrystals were precipitated with hexane by centrifugation.

For the synthesis of $\mathrm{Mn}_{3} \mathrm{O}_{4}$ nanocrystals with edge length of $15 \mathrm{~nm}, 4.9 \mathrm{~g}$ manganese (II) acetate tetrahydrate (Aldrich), $53.4 \mathrm{~g}$ oleylamine (Acros) and $2.8 \mathrm{~g}$ oleic acid (Aldrich) were dissolved in $300 \mathrm{ml}$ of xylene (Aldrich). The mixture was stirred at room temperature over $3 \mathrm{~h}$ in a three-necked 1,000-ml flask. The reaction mixture was slowly heated to $90^{\circ} \mathrm{C}$ in air under magnetic stirring, and after it reached $90^{\circ} \mathrm{C}, 7 \mathrm{ml}$ of deionized water was rapidly injected into the reaction solution. The solution was aged at $90^{\circ} \mathrm{C}$ in air for $1.5 \mathrm{~h}$. The solution was then cooled to room temperature and washed with ethanol. The $\mathrm{Mn}_{3} \mathrm{O}_{4}$ nanocrystals were retrieved by centrifugation. The ligand exchange procedure using $\mathrm{NOBF}_{4}$ was the same as that described for the 11-nm-sized nanoparticles. 
For the synthesis of $\mathrm{Mn}_{3} \mathrm{O}_{4}$ nanocrystals with edge length of $18 \mathrm{~nm}, 4.9 \mathrm{~g}$ manganese (II) acetate tetrahydrate (Aldrich), $53.4 \mathrm{~g}$ oleylamine (Acros) and $2.8 \mathrm{~g}$ oleic acid (Aldrich) were dissolved in $300 \mathrm{ml}$ of xylene (Aldrich). The mixture was stirred at room temperature over $3 \mathrm{~h}$ in a three-necked 1,000-ml flask. The reaction mixture was slowly heated to $100^{\circ} \mathrm{C}$ in air under magnetic stirring, and after it reached $100^{\circ} \mathrm{C}, 8 \mathrm{ml}$ of deionized water was rapidly injected into the reaction solution. Then, the solution was aged at $90^{\circ} \mathrm{C}$ in air for $1.5 \mathrm{~h}$. Thereafter, the solution was cooled to room temperature and washed with ethanol. The $\mathrm{Mn}_{3} \mathrm{O}_{4}$ nanocrystals were retrieved by centrifugation. The ligand exchange procedure using $\mathrm{NOBF}_{4}$ was the same as that described for the

11-nm-sized nanoparticles.

For the synthesis of $\mathrm{Mn}_{3} \mathrm{O}_{4}$ nanocrystals with edge length of $20 \mathrm{~nm}, 4.9 \mathrm{~g}$ manganese (II) acetate tetrahydrate (Aldrich), $13.4 \mathrm{~g}$ oleylamine (Acros) and $2.8 \mathrm{~g}$ oleic acid (Aldrich) were dissolved in $300 \mathrm{ml}$ of xylene (Aldrich). The mixture was stirred at room temperature for $3 \mathrm{~h}$ in a three-necked 1,000-ml flask. The reaction mixture was slowly heated to $105^{\circ} \mathrm{C}$ in air under magnetic stirring, and after it reached $105^{\circ} \mathrm{C}, 20 \mathrm{ml}$ of deionized water was rapidly injected into the reaction solution. Then, the solution was aged at $90^{\circ} \mathrm{C}$ in air for $1.5 \mathrm{~h}$. Thereafter, the solution was cooled to room temperature and washed with ethanol. The $\mathrm{Mn}_{3} \mathrm{O}_{4}$ nanocrystals were retrieved by centrifugation. The ligand exchange procedure using $\mathrm{NOBF}_{4}$ was the same as that described for the 11-nm-sized nanoparticles.

Synthesis of LiF-C/Mn $\mathbf{M n}_{4}$ mixture electrode. $\mathrm{LiF}-\mathrm{C} / \mathrm{Mn}_{3} \mathrm{O}_{4}(6,9,11,15,18$, $20 \mathrm{~nm}$ ) mixture electrodes were fabricated using the following sequence. In total, $70 \mathrm{wt} \%$ of the electrode was composed by a mixture of the $\mathrm{LiF}-\mathrm{C}$ nanocomposite and $\mathrm{Mn}_{3} \mathrm{O}_{4}$ nanocrystals $(\mathrm{Li} / \mathrm{Mn}=1 / 1 \mathrm{~m} / \mathrm{m}$ ). The $\mathrm{LiF}-\mathrm{C}$ nanocomposite was synthesized using planetary ball milling (Pulverisette 5; Fritsch). As-received LiF (Sigma-Aldrich) and F-free graphite (Alfa Aesar) were used without further purification. $\mathrm{MnO}$ and graphite were inserted into the same container $(\mathrm{LiF} / \mathrm{C}$ : 1/1, w/w) with an Ar atmosphere in a glove box. The powders were mixed in the container at 400 r.p.m. for $48 \mathrm{~h}$ without rest time. In addition, $20 \mathrm{wt} \%$ carbon black (Super-P; Timcal) and $10 \mathrm{wt} \%$ F-free binder (polyacrylonitrile, PAN) dissolved in $N$-methyl-1,2,-pyrrolidone (NMP, 99.5\%; Sigma-Aldrich) were cast onto aluminium foil using a doctor blade. The NMP was evaporated overnight at $25^{\circ} \mathrm{C}$ in a vacuum oven. The final portion of active material $\left(\mathrm{LiF} / \mathrm{Mn}_{3} \mathrm{O}_{4}\right.$ except carbon) was set as $55.8 \mathrm{wt} \%$.

Electrochemical measurements. Test electrodes were fabricated with the following sequence. A slurry of $70 \mathrm{wt} \% \mathrm{LiF}-\mathrm{MO}$ nanocomposite, $20 \mathrm{wt} \%$ carbon black (Super-P; Timcal), and $10 \mathrm{wt} \%$ polyvinylidene fluoride (PVDF) binder dissolved in $N$-methyl-1,2,-pyrrolidone (NMP, 99.5\%; Sigma-Aldrich) was cast onto aluminium foils using a doctor blade. The NMP was evaporated overnight at $25^{\circ} \mathrm{C}$ in a vacuum oven. Coin cells (CR2032; Hohsen) were assembled with the $\mathrm{LiF}-\mathrm{MnO}$ nanocomposite electrode, lithium counter electrode, a separator washed with acetone (GF/F Filter; Whatman), and a $1 \mathrm{M}$ solution of $\mathrm{LiPF}_{6}$ in a mixture of ethyl carbonate/dimethyl carbonate (EC/DMC, 1:1, v/v) in an argon-filled glove box. For XPS measurement, a fluorine-free binder (polyacrylonitrile, PAN) and a $1 \mathrm{M}$ solution of $\mathrm{LiClO}_{4}$ in $\mathrm{EC} / \mathrm{DMC}(1: 1, \mathrm{v} / \mathrm{v})$ were used. To evaluate the properties of the LiF-MO nanocomposite, galvanostatic charge/discharge profiles were measured over voltage ranges of $1.5-4.8 \mathrm{~V}$ with a potentiogalvanostat (WBCS 3000; WonA Tech) at room temperature. $\mathrm{LiF}-\mathrm{C} / \mathrm{Mn}_{3} \mathrm{O}_{4}$ mixture electrode was tested over voltage ranges of 2.0-4.8 V. The first charge process was performed under the constant current and constant voltage $(\mathrm{CC} / \mathrm{CV})$ mode to $4.8 \mathrm{~V}$ under $20 \mathrm{~mA} \mathrm{~g}^{-1}$ at room temperature and maintained until the current density decreased below $10 \mathrm{~mA} \mathrm{~g}^{-1}$. The capacity was calculated by dividing the absolute capacity by the weight of active material including $\mathrm{LiF}$ and $\mathrm{MO}(\mathrm{M}=\mathrm{Mn}, \mathrm{Fe}, \mathrm{Co})$. $\mathrm{CV}$ measurements were obtained after the first charge using the same protocol as for the galvanostatic charge. The voltage was scanned from 4.8 to $1.5 \mathrm{~V}$ as the scan rate was changed from 0.075 to $1.00 \mathrm{mV} \mathrm{s}^{-1}$. The peak currents at $3.75 \mathrm{~V}$ and $2.5 \mathrm{~V}$ redox reaction were plotted as scan rate and analysed by linear fit in log scale. The adjusted $R^{2}$ value of $3.75 \mathrm{~V}$ was the same as 0.9995 at cathodic and anodic reaction. The values of $2.5 \mathrm{~V}$ were 0.9994 and 0.9952 at cathodic and anodic reaction respectively. The standard errors of $3.75 \mathrm{~V}$ were 0.00949 and 0.00973 at cathodic and anodic reaction. In the case of $2.5 \mathrm{~V}$, the standard errors were 0.04039 and 0.01579 for each. The full cell was assembled into a coin-type cell with a $\mathrm{Li}_{4} \mathrm{Ti}_{5} \mathrm{O}_{12}$ electrode as the negative electrode and $\mathrm{LiF}-\mathrm{MnO}$ nanocomposite as the positive electrode. The cell was tested in the voltage range $0.01-3.3 \mathrm{~V}$ at a current rate of $20 \mathrm{~mA} \mathrm{~g}^{-1}$ based on the weight of the positive electrode.

$\mathrm{X}$-ray absorption spectroscopy and diffraction analysis. For in situ absorption spectroscopy (XAS) experiments, holed coin cells sealed using epoxy with Kapton film were used. The current rate was fixed at $20 \mathrm{~mA} \mathrm{~g}^{-1}$, and the charge discharge protocol followed the same conditions as those used in the galvanostatic experiments. In situ XAS was performed at the 7D XAFS and 10C wide XAFS beamlines at the Pohang Light Source-II (PLS-II) using the Si(111) double-crystal monochromator. The beam intensity was reduced by $20 \%-30 \%$ to minimize high-order harmonics. The $\mathrm{Mn}$ and Fe K-edge spectra of $\mathrm{LiF}-\mathrm{MO}(\mathrm{M}=\mathrm{Mn}, \mathrm{Fe})$ electrodes were recorded in transmission mode at room temperature. The storage ring was operated at $2.5 \mathrm{GeV}$ with a ring current of $100-150 \mathrm{~mA}$. Reference spectra of the Mn and Fe metals were collected simultaneously using Mn and Fe metal foils. The XAS data were analysed using the Athena program, and the spectral energies were calibrated using the first inflection points in the $\mathrm{Mn}$ and $\mathrm{Fe}$ metal foil spectra. The extracted extended X-ray absorption fine structure (EXAFS) signal, $\chi(\mathrm{k})$, was weighted by $k^{3}$ to emphasize the high-energy oscillations and then Fourier-transformed in a $k$ range from 2.0 to $11.5 \AA^{-1}$ for the $\mathrm{Mn}$ and 2.0 to $11.8 \AA^{-1}$ for the Fe K-edge using a Hanning window to obtain the magnitude plots of the EXAFS spectra in $R$-space $(\AA)$. Data pre-processing operations such as deglitching, energy calibration, normalization, and least-squares fitting with theory were performed as described in ref. 33 using IFEFFIT $^{34}$, which used the FEFF code ${ }^{35}$. Ex situ XRD patterns for the LiF-MnO electrode were collected on the 5A XRD beamline at PLS-II. Samples were prepared by subdividing points in the charge/discharge cycles. Electrodes were collected by disassembling the coin cell after achieving the set capacity. Collected electrodes were washed using diethyl carbonate and dried in a dry room. Samples were sealed using Kapton tape and packed in an aluminium pouch to prevent contamination. The wavelength of the X-ray beam was $0.7653 \AA$, and XRD patterns were recorded as a set of circles on a Mar 345-image plate detector in the transmission mode for about $1 \mathrm{~min}$ of exposure time. The total recording time was about $2.6 \mathrm{~min}$, including the scanning time of the image plate and transfer time of spectral information. The $2 \theta$ angles of all the XRD patterns presented in this article have been recalculated to corresponding angles for $\lambda=1.54 \AA$, which is the wavelength of the conventional X-ray tube source with $\mathrm{Cu} \mathrm{K} \alpha$ radiation for easy comparison with other published results. High-resolution powder XRD data of $\mathrm{Mn}_{3} \mathrm{O}_{4}$ nanoparticles were obtained from Beamline 9B at Pohang Accelerator Laboratory (PAL), Republic of Korea. The data were collected over a $2 \theta$ range of $10^{\circ}-130^{\circ}$ with a step size of $0.01^{\circ}$, step time of $6 \mathrm{~s}$, and wavelength of $\lambda=1.4865 \AA$.

Near-edge X-ray absorption fine-structure spectroscopy. NEXAFS spectra of the $\mathrm{Mn} \mathrm{L}_{3,2}$-edge and the F K-edge were taken at the 10D KIST bending magnet beamline at the Pohang Light Source-II (PLS-II). All of the measurements were performed at room temperature and the spectra were collected in a total electron yield mode under a base pressure of $3 \times 10^{-10}$ torr. The spectra were normalized to the incident photon flux, and the energy resolution was $0.1 \mathrm{eV}$.

Ex situ X-ray photoelectron spectroscopy. Half-cells of the LiF-MO ( $\mathrm{M}=\mathrm{Mn}$, $\mathrm{Fe}$ ) nanocomposites were assembled and electrochemically cycled. The charged electrode was collected by disassembling the coin cell charged to $4.8 \mathrm{~V}$, and the discharged electrode was collected from the coin cell discharged to $1.5 \mathrm{~V}$ after being charged to $4.8 \mathrm{~V}$. The collected electrodes were washed using DMC and dried. The chemical states and composition of the collected electrodes were identified by X-ray photoelectron spectroscopy (Thermo VG Scientific).

TEM and STEM-EELS measurements. For ex situ analysis, electrodes at different states of charge in half-cells were disassembled in a glove box and washed using DMC before drying. The electrodes detached from the aluminium current collector were immersed in DMC and dispersed using sonication. The TEM sample grid was covered by DMC to minimize any exposure to air during the sample transfer and kept in a vacuum before being transferred to the transmission electron microscope $\mathrm{e}^{36}$.

STEM-EELS measurements were performed using a cold-field-emission-type Cs-corrected JEM-ARM200F equipped with an EELS detector (965 GIF Quantum ER) installed at the National Center for Inter-university Research Facilities (NCIRF) at Seoul National University. High-resolution annular dark-field images were collected with $0.1-\mathrm{nm}$ spatial resolution. TEM was performed at $80 \mathrm{kV}$. For STEM-EELS elemental mapping, the energy dispersion was set as $0.25 \mathrm{eV}$ per channel. The full-width at half-maximum of the zero-loss peak in vacuum was $1.0 \mathrm{eV}$. The convergence and collection semiangles were 19 and $52.8 \mathrm{mrad}$, respectively. TEM and energy-filtered TEM (EFTEM) images of the as-synthesized $\mathrm{Mn}_{3} \mathrm{O}_{4}$ nanoparticles and $\mathrm{LiF}-\mathrm{C} / \mathrm{Mn}_{3} \mathrm{O}_{4}$ electrode were captured using a JEOL-2100F at $200 \mathrm{kV}$.

Data availability. The data that support the plots within this paper and other findings of this study are available from the corresponding author on request.

Received 16 July 2016; accepted 13 December 2016; published 23 January 2017

\section{References}

1. Larcher, D. \& Tarascon, J. M. Towards greener and more sustainable batteries for electrical energy storage. Nat. Chem. 7, 19-29 (2015). 
2. Sathiya, M. et al. Reversible anionic redox chemistry in high-capacity layered-oxide electrodes. Nat. Mater. 12, 827-835 (2013).

3. Lee, J. et al. Unlocking the potential of cation-disordered oxides for rechargeable lithium batteries. Science 343, 519-522 (2014).

4. Kim, S.-W. et al. Energy storage in composites of a redox couple host and a lithium ion host. Nano Today 7, 168-173 (2012).

5. Tarascon, J. M. \& Armand, M. Issues and challenges facing rechargeable lithium batteries. Nature 414, 359-367 (2001)

6. Tarascon, J. M., Wang, E., Shokoohi, F. K., McKinnon, W. R. \& Colson, S The spinel phase of $\mathrm{LiMn}_{2} \mathrm{O}_{4}$ as a cathode in secondary lithium cells. J. Electrochem. Soc. 138, 2859-2864 (1991)

7. Padhi, A. K., Nanjundaswamy, K. S. \& Goodenough, J. B. Phospho-olivines as positive-electrode materials for rechargeable lithium batteries. J. Electrochem. Soc. 144, 1188-1194 (1997).

8. Mizushima, K., Jones, P. C., Wiseman, P. J. \& Goodenough, J. B. $\mathrm{Li}_{x} \mathrm{CoO}_{2}$ $(0<x<-1)$ : a new cathode material for batteries of high energy density. Mater. Res. Bull. 15, 783-789 (1980).

9. Poizot, P., Laruelle, S., Grugeon, S., Dupont, L. \& Tarascon, J. M. Nano-sized transition-metal oxides as negative-electrode materials for lithium-ion batteries. Nature 407, 496-499 (2000).

10. de Faria, D. L. A., Venâncio Silva, S. \& de Oliveira, M. T. Raman microspectroscopy of some iron oxides and oxyhydroxides. J. Raman Spectrosc. 28, 873-878 (1997)

11. Fang, X. et al. Electrode reactions of manganese oxides for secondary lithium batteries. Electrochem. Commun. 12, 1520-1523 (2010).

12. Sun, B., Chen, Z., Kim, H.-S., Ahn, H. \& Wang, G. MnO/C core-shell nanorod as high capacity anode materials for lithium-ion batteries. J. Power Sources 196, 3346-3349 (2011).

13. Gao, M. et al. FeO/C anode materials of high capacity and cycle stability for lithium-ion batteries synthesized by carbothermal reduction. J. Alloys Compd. 565, 97-103 (2013)

14. Dimov, N., Kitajou, A., Hori, H., Kobayashi, E. \& Okada, S. Electrochemical splitting of LiF: a new approach to lithium-ion battery materials. ECS Trans. $\mathbf{5 8}$ 87-99 (2014)

15. Augustyn, V. et al. High-rate electrochemical energy storage through Li+ intercalation pseudocapacitance. Nat. Mater. 12, 518-522 (2013).

16. Wang, J., Polleux, J., Lim, J. \& Dunn, B. Pseudocapacitive contributions to electrochemical energy storage in $\mathrm{TiO}_{2}$ (anatase) nanoparticles. J. Phys. Chem. C 111, 14925-14931 (2007)

17. Brezesinski, T., Wang, J., Polleux, J., Dunn, B. \& Tolbert, S. H. Templated nanocrystal-based porous $\mathrm{TiO}_{2}$ films for next-generation electrochemical capacitors. J. Am. Chem. Soc. 131, 1802-1809 (2009).

18. Dunn, B., Kamath, H. \& Tarascon, J.-M. Electrical energy storage for the grid: a battery of choices. Science 334, 928-935 (2011).

19. Gilbert, B. et al. Multiple scattering calculations of bonding and X-ray absorption spectroscopy of manganese oxides. J. Phys. Chem. A 107, 2839-2847 (2003).

20. Qiao, R., Chin, T., Harris, S. J., Yan, S. \& Yang, W. Spectroscopic fingerprints of valence and spin states in manganese oxides and fluorides. Curr. Appl. Phys. 13, 544-548 (2013).

21. Augustyn, V., Simon, P. \& Dunn, B. Pseudocapacitive oxide materials for high-rate electrochemical energy storage. Energy Environ. Sci. 7, 1597-1614 (2014).

22. Kim, S.-W. et al. Structure stabilization by mixed anions in oxyfluoride cathodes for high-energy lithium batteries. ACS Nano 9, 10076-10084 (2015)

23. Wang, X.-L. et al. Amorphous hierarchical porous GeOx as high-capacity anodes for Li ion batteries with very long cycling life. J. Am. Chem. Soc. 133, 20692-20695 (2011).

24. Yamakawa, N., Jiang, M., Key, B. \& Grey, C. P. Identifying the local structures formed during lithiation of the conversion material, iron fluoride, in a Li ion battery: a solid-state NMR, X-ray diffraction, and pair distribution function analysis study. J. Am. Chem. Soc. 131, 10525-10536 (2009).

25. Pereira, N., Badway, F., Wartelsky, M., Gunn, S. \& Amatucci, G. G. Iron oxyfluorides as high capacity cathode materials for lithium batteries. J. Electrochem. Soc. 156, A407-A416 (2009).

26. Wiaderek, K. M. et al. Comprehensive insights into the structural and chemical changes in mixed-anion FeOF electrodes by using Operando PDF and NMR spectroscopy. J. Am. Chem. Soc. 135, 4070-4078 (2013).

27. Choi, S. \& Manthiram, A. Factors influencing the layered to spinel-like phase transition in layered oxide cathodes. J. Electrochem. Soc. 149, A1157-A1163 (2002).

28. Kim, H. et al. Multicomponent effects on the crystal structures and electrochemical properties of spinel-structured $\mathrm{M}_{3} \mathrm{O}_{4}(\mathrm{M}=\mathrm{Fe}, \mathrm{Mn}, \mathrm{Co})$ anodes in lithium rechargeable batteries. Chem. Mater. 24, 720-725 (2012).

29. Sina, M., Pereira, N., Amatucci, G. G. \& Cosandey, F. Microstructural evolution of iron oxyfluoride/carbon nanocomposites upon electrochemical cycling. J. Phys. Chem. C 120, 13375-13383 (2016).

30. Oh, M. H. et al. Galvanic replacement reactions in metal oxide nanocrystals. Science 340, 964-968 (2013).

31. Vlasse, M., Massies, J. C. \& Demazeau, G. The refinement of the crystal structure of iron oxyfluoride, FeOF. J. Solid State Chem. 8, 109-113 (1973).

32. Dong, A. et al. A generalized ligand-exchange strategy enabling sequential surface functionalization of colloidal nanocrystals. J. Am. Chem. Soc. 133 , 998-1006 (2011).

33. Kelly, S., Hesterberg, D. \& Ravel, B. Analysis of soils and minerals using X-ray absorption spectroscopy. Methods Soil Anal. 5, 387-463 (2008).

34. Ravel, B. \& Newville, M. ATHENA, ARTEMIS, HEPHAESTUS: data analysis for X-ray absorption spectroscopy using IFEFFIT. J. Synchrotron Radiat. 12, 537-541 (2005).

35. Rehr, J. J. \& Albers, R. Theoretical approaches to X-ray absorption fine structure. Rev. Mod. Phys. 72, 621-654 (2000).

36. Lee, S.-Y. et al. Unveiling origin of additional capacity of $\mathrm{SnO}_{2}$ anode in lithium-ion batteries by realistic ex situ TEM analysis. Nano Energy 19, 234-245 (2016)

\section{Acknowledgements}

This work was supported by Samsung Research Funding Centre of Samsung Electronics under Project Number SRFC-TA1403-03. Also, this work was supported by Project Code (IBS-R006-G1). S.-K.J., H.K., K.-Y.P. and K.K. are grateful for the financial support from IBS

\section{Author contributions}

S.-K.J. conceived the research and carried out the synthesis, electrochemical test, and characterization. H.K. performed the overall X-ray absorption spectroscopy experiment and analyses. M.G.C. synthesized the $\mathrm{Mn}_{3} \mathrm{O}_{4}$ nanoparticle and performed TEM work for the $\mathrm{LiF}-\mathrm{C} / \mathrm{Mn}_{3} \mathrm{O}_{4}$ system. S.-P.C. conducted TEM and STEM-EELS measurements for the $\mathrm{LiF}-\mathrm{MnO}$ nanocomposite. B.L. and G.Y. conducted the DFT calculations and developed the theoretical model. W.-S.Y. and K.K. supervised the overall research. All authors discussed the experiments and final manuscript.

\section{Additional information}

Supplementary information is available for this paper.

Reprints and permissions information is available at www.nature.com/reprints. Correspondence and requests for materials should be addressed to W.-S.Y. or K.K.

How to cite this article: Jung, S.-K. et al. Lithium-free transition metal monoxides for positive electrodes in lithium-ion batteries. Nat. Energy 2, 16208 (2017).

\section{Competing interests}

The authors declare no competing financial interests. 